Fecha de recepción: 09-10-2017

Fecha de aceptación: 29-01-2018

Link para este artículo: http://dx.doi.org/10.14198/ALEUA.2018.29-30.05

Puede citar este artículo como:

Urgel Cantalejo, Juan Luis, «Agustín González y sus comienzos como actor en el TEU», Anales de

Literatura Española, n. ${ }^{\circ}$ 29-30 (2018), pp. 119-132.

\title{
AGUSTÍN GONZÁLEZ Y SUS COMIENZOS COMO ACTOR EN EL TEU
}

\author{
JUAN LUIS URGEL CANTALEJO \\ ESAD de Extremadura
}

\section{Resumen}

El TEU supuso para Agustín González el momento crucial de su carrera. Sin él, seguramente no habría podido dedicarse profesionalmente a la interpretación. Pero también significó para él un lugar de formación artística, reflexión y acción social, y un vivero creativo donde pudo relacionarse con las personalidades más vanguardistas del teatro de la época. Gracias al TEU, Agustín González colaboró en dos estrenos de importancia capital para la historia del teatro español de la posguerra: Tres sombreros de copa y Escuadra hacia la muerte, e intervino en la realidad cultural del momento, no de forma incidental, sino con un papel relevante.

Palabras clave: Teatro, Actor, Agustín González, Teatro Español Universitario, Tres sombreros de copa, Escuadra hacia la muerte.

\begin{abstract}
TEU was the turning point in Agustín González's career. Without it, he wouldn't have been able to dedicate himself to professional acting. But it also meant for him a place for the artistic education, the reflexion and social action, as well as a creative environment where he could meet the most avant-garde personalities of his time's theatre. Thanks to TEU, Agustín González worked in two plays of the highest importance for the Spanish post-war theatre: Tres sombreros de copa and Escuadra hacia la muerte, and he took a place in his period's cultural reality, not incidentally, but with a relevant role.
\end{abstract}

Keywords: Theatre, Actor, Agustín González, Teatro Español Universitario, Tres sombreros de copa, Escuadra hacia la muerte. 
La labor de Agustín González en el TEU de Madrid abarca los tres años que van desde 1952 a 1954. Como veremos, es un representante de primer orden de lo que Juan Antonio Hormigón denomina «la primera etapa» (1974) del TEU, de 1940 a 1955. Si bien Hormigón señala «los sucesos acaecidos en la Universidad de Madrid en 1956» (1974) como desencadenante de una especie de edad de oro de los TEUs, y sin menospreciar la importancia que esos hechos tuvieron para la comunidad universitaria y la sociedad madrileña en general, los estrenos de Tres sombreros de copa (1952) y Escuadra hacia la muerte (1953) supusieron un impacto mayor, no sólo para el TEU de Madrid, sino para la historia del teatro español del siglo XX.

Así pues, se puede decir que la evolución de Agustín González en el TEU de Madrid es una muestra de la evolución del propio TEU, que pasa de la escenificación del teatro clásico a la de los autores contemporáneos más alejados de la ideología franquista, de la universidad al teatro independiente; y que supone una renovación del panorama teatral desde el mundo universitario.

Agustín González trabajaría esos años con Salvador Salazar, primero, y Gustavo Pérez Puig, después, como directores del TEU de Madrid y del Teatro Popular Universitario (TPU), creado por Salazar. Venía de una familia con varios miembros artistas. Según él mismo cuenta, su abuela era descendiente directa de Calderón de la Barca, su hermano era el bajista de Los Brincos, y tenía un primo lejano torero (Millás, 2005: 23-25). Una tía suya fue dama joven de la compañía de Margarita Xirgu en América. Así lo cuenta: «Fue durante aquel viaje por América, mientras se encontraban en Chile, cuando mi tía Antoñita y José Jordá se casaron. Sus padrinos de boda fueron Margarita Xirgu y el director de la compañía, Cipriano Rivas Cherif» (Millás, 2005: 25). Pero a su padre le ilusionaba la idea de que fuera arquitecto. «Empecé a estudiar Perito industrial, aparejador, carreras más o menos dentro de esta línea, sobre todo con la idea de darle una satisfacción a mi padre, pero aquello no me iba, no estudiaba, me aburría en las clases» (Millás, 2005: 34). Probó después con Filosofía y Letras, pero desde el comienzo le atraía más el teatro, de modo que en cuanto tuvo ocasión abandonó los estudios para dedicarse en exclusiva a la interpretación. De hecho, antes de encontrarse con Salazar y Pérez Puig, ya había intervenido en varias representaciones de aficionados en Huarte y Cía. (la empresa donde trabajaba su padre) y en la propia universidad, donde fundó la compañía La Diabla junto a Rafael y Emiliano Redondo y Lauro Olmo, compañeros que, como él, darían luego el salto al teatro profesional. Esta compañía amateur del ámbito universitario representaba en el teatro del Parque Móvil de los Ministerios (Millás, 2005: 35-36). 


\section{2: de los comienzos a la consagración}

Pero el comienzo de Agustín González en el Teatro Español Universitario de Madrid no se daría hasta el 29 de marzo de 1952, en el TEU de la Facultad de Letras, con veintidós años y el estreno de La verdad de cada cual de Luigi Pirandello en el Paraninfo de la Facultad de Letras de la Universidad de Madrid. ${ }^{1}$ Posteriormente, tuvo lugar el estreno en programa triple de Las palabras en la arena de Buero Vallejo, Un caso curioso de Pablo Martí Zaro y Yo, Eva de Alfonso Paso, dirigido por el propio González. ${ }^{2}$ Conforme a las circunstancias del momento, este espectáculo se estrenó en función única el miércoles 2 de abril de 1952 en el teatro Beatriz de Madrid. Era la primera vez que González actuaba en un teatro profesional (aunque no como profesional), y lo hacía con su compañero de La Diabla Rafael Redondo y Juanjo Menéndez, quien también se estrenaba en el TEU con este montaje. El 15 de mayo, con el TEU de la Facultad de Letras y en el Paraninfo de la misma, estrenó El caballero de Olmedo de Lope de Vega, coincidiendo por primera vez con Fernando Guillén. Y más adelante, Todos los hijos de Dios tienen alas de Eugene O'Neill, donde también colaboró con Guillén.

Con el TEU de Madrid y bajo la dirección de Salvador Salazar, representó La farsa de Micer Patelin, el primer montaje de éste como director del TEU. Con él, Salazar relevaba a Modesto Higueras en la dirección del TEU de Madrid, labor que había desempeñado durante diez años, desde su fundación en 1940. Ardua tarea la de sustituir a un director que había conformado durante tanto tiempo el TEU logrando que se alejara de ser una herramienta propagandística del régimen para acercarse más al espíritu de La Barraca de Federico García Lorca, con su vocación renovadora y popularizante. Y Salazar no sólo continuó en esta línea, sino que fundó el Teatro Popular Universitario (TPU), en el que también colaborará Agustín González. Sobre el TPU como sección del TEU, Alfonso Sastre manifestó que «es la expresión de un momento en el que todo lo que se ha hecho resulta insuficiente. Hay que salir no sólo a la calle de la ciudad - desde los limitados y asépticos recintos universitarios -, sino al campo de España» (1954: 173-174).

1. Para los estrenos de La verdad de cada cual, El caballero de Olmedo, Todos los hijos de Dios tienen alas y El momento de tu vida la información ha sido facilitada por Pilar Palomo, compañera de reparto en todos ellos de Agustín González, a quien estoy profundamente agradecido.

2. Para ésta y las demás referencias a estrenos de espectáculos (no siendo las facilitadas por Pilar Palomo), la información está extraída de la base de datos del Centro de Documentación Teatral. <http://teatro.es/profesionales/agustin-gonzalez-agustin-gonzalez-martinez-2725/estrenos> [consulta: 9 marzo 2017]. 
Conforme a las circunstancias del momento, La farsa de Micer Patelin se estrenó en función única el lunes 22 de septiembre de 1952 en el teatro María Guerrero de Madrid, coincidiendo con el día de descanso de la compañía que estaba en cartel. Con este clásico francés del siglo XV, Agustín González y Salvador Salazar colaboraron con Karen Taft y José María Prada, entre otros. La ocasión de poder estrenar en un teatro de tal importancia, González la cifra en la personalidad del director del María Guerrero, a la sazón Luis Escobar, falangista; y así lo manifiesta:

La suerte fue que la dirección del María Guerrero cayera en sus manos porque hizo una programación, en la década de los cuarenta, fascinante, mostrando obras extraordinarias que, de no haber sido por él, no se hubieran conocido. Además, consiguió crear una compañía por donde pasaron los mejores actores de aquel entonces y otros que, posteriormente, fueron muy considerados, como es el caso de Rafael Alonso, José María Rodero o Adolfo Marsillach (Millás, 2005: 30).

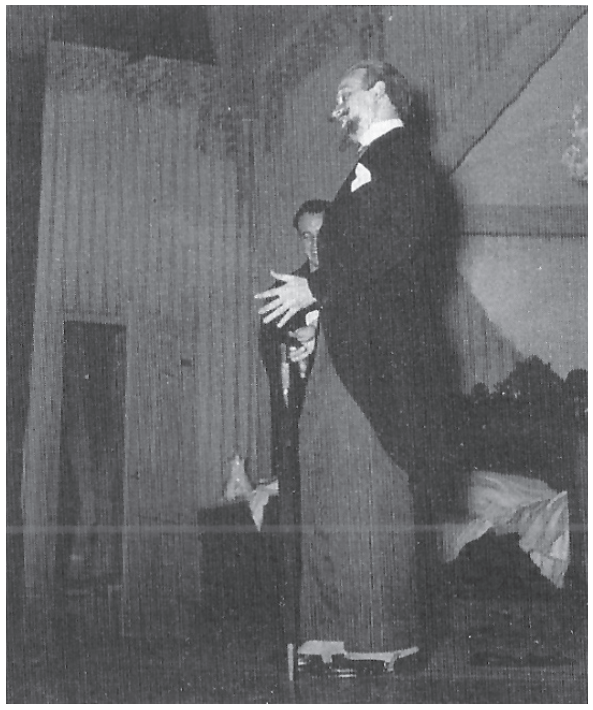

Como decíamos, uno de los dos acontecimientos fundamentales de la historia del teatro español del siglo xx en que participó Agustín González fue el estreno, en principio en función única, de Tres sombreros de copa, de Miguel Mihura, el lunes 24 de noviembre de 1952 en el Teatro Español de Madrid, bajo la dirección de Gustavo Pérez Puig. Además, significó el momento más decisivo en la carrera de Agustín González por permitirle dar el salto al mundo profesional, pues, como él mismo reconocía, a partir de ese momento siempre vivió de su trabajo como actor (Millás, 2005: 37). Su biógrafa profundiza en la misma línea: 
El estreno de la obra de Miguel Mihura, Tres sombreros de copa, que tuvo lugar en el Teatro Español de Madrid el 24 de noviembre de 1952, es una constante en sus recuerdos ya que de ahí arranca su carrera profesional. Una y otra vez se refiere a este suceso como si quisiera grabarlo en mi memoria con la misma fuerza que, al parecer, permanece en la suya. A la fecha y al título asocia de inmediato el nombre de sus compañeros, empezando por el director Gustavo Pérez Puig para continuar con Juanjo Menéndez, Fernando Guillén, Pepe Cerro o Rafael Martín Peña. Insiste, de mil maneras, en la importancia que tuvo la representación de una obra escrita veinte años atrás, que nadie se había atrevido a estrenar y que rompió los moldes de un teatro que olía a rancio, a adocenado y demasiado convencional. Cuenta emocionado cómo aquel grupo de jóvenes del que formaba parte, fue capaz de trasladar al público una ráfaga de creatividad, frescura y alegría, dando vida a una criatura diferente, incomprendida durante años y perfectamente encuadrada dentro del movimiento surrealista español. Pone especial énfasis al señalar que se adelantó en su estilo, nada menos que diecisiete años, a La cantante calva de Ionesco y a otros autores del teatro del absurdo. ¡NOSOTROS ENTENDIMOS PERFECTAMENTE A MIHURA!

Ya sé que no podemos estar hablando siempre de Tres sombreros de copa, dice en una de nuestras charlas, pero para mí, su estreno, junto con el de Escuadra hacia la muerte, de Alfonso Sastre, supone la aparición de un fenómeno especialmente valioso para estudiar un momento histórico muy importante que tuve ocasión de vivir. Entonces, cuando yo encarnaba a un personaje tan querido como don Sacramento, el teatro era un elemento vivo y de combate, era un arma, cosa que ahora ha dejado de ser (2005: 77-78).

Jorge Rodríguez Padrón, en su edición para Cátedra de la obra que lanzó al éxito a Agustín González, coincide en remarcar la comprensión del texto que tenía el grupo universitario que llevó a cabo su estreno y el éxito que consiguió, brindándonos también un valioso testimonio de la opinión del autor al respecto, así como de lo inesperado que resultó:

Cuando en 1952, el TEU de Madrid, bajo la dirección de Gustavo Pérez Puig, decide poner en escena Tres sombreros de copa ha de vencer una durísima resistencia de parte del autor, quien no sólo ha olvidado la obra, sino que no desea ya estrenarla nunca. La habilidad y el entusiasmo de Pérez Puig fueron decisivos, y después de leer la obra, entusiasmado por el descubrimiento, se entrevista con Mihura, quien por tratarse de un grupo universitario, porque se daría en función única y, sobre todo, por la decisión, la sinceridad y el ímpetu que ponía en todo cuanto decía el por entonces director del TEU (son palabras del propio Mihura), acaba cediendo y da la correspondiente autorización. La sorpresa de Mihura se produjo cuando supo, el mismo día del estreno, que la función se daría en el Teatro Español; cuando ve el teatro lleno de público y cómo la crítica prestó inusitado interés al estreno; más aún, cuando comprueba cómo, al fin, alguien entendía la obra hasta el punto de quedar encantado «de cómo aquellos jóvenes interpretaban la comedia. Y de la forma en que estaba 
montada». Todo esto ocurría la noche del 24 de noviembre de 1952. Y tras el éxito, «como no esperaba nada de esto me quedé abrumado y derrumbado. Y con una alegría exterior que me era imposible disimular. Pero lo más extraño de todo, lo más dramático, es que me encontré viejo [...] este éxito me consagraba como autor, pero [...] había llegado demasiado tarde». Mihura ganaría ese año, y con esta obra, su primer Premio Nacional de Teatro, y Tres sombreros de copa quedó desde entonces como una de las obras integrantes de la trilogía fundamental en nuestro teatro de posguerra (aunque se escribiese cuatro años antes del comienzo de la contienda civil), junto a Historia de una escalera, de Buero Vallejo y Escuadra hacia la muerte, de Alfonso Sastre (1997: 22-23).

Así pues, lo que en principio era una representación universitaria, acabó significando la lanzadera al éxito de su autor, su director y parte de su elenco. Al día siguiente del estreno, aparecieron artículos en Arriba, ABC, Informaciones, Madrid y El Alcázar, y en días posteriores, en Destino, Ateneo, y Arriba nuevamente. El espectáculo tuvo tanta aceptación que la función se repitió el lunes siguiente (algo inaudito para el TEU, puesto que el sistema de representación única suponía un efectivo mecanismo de censura para el régimen), y así continuó hasta que el actor Luis Prendes se hizo con los derechos de la comedia para incorporarla al repertorio de su compañía y representarla en el teatro Beatriz en menos de un mes desde la fecha de su estreno. En el Beatriz, Luis Prendes contó con Pérez Puig, José María Prada y Agustín González del equipo original del TEU, y la repercusión siguió aumentando hasta que, en la décima representación, en la escena de la fiesta en la habitación, aparecieron figurantes de la categoría de la periodista Josefina Carabias, Miguel Pérez Ferrero, Luis Alonso, Pepe Franco, Fernán-Gómez, los Ozores, Edgard Neville, María Asquerino, Emma Penella, Virginia de Matos, Luis Escobar... Así lo cuenta el propio Agustín González:

Lo cierto es que Luis, a la vista del éxito que tuvo el estreno de Tres sombreros de copa, decidió incorporarla a su temporada, para lo cual, nos contrató a algunos de los que habíamos estrenado la función. No recuerdo si antes había realizado algún papel que me hubiera proporcionado dinero, pero en aquella ocasión cobré mi trabajo y tuve, por primera vez, un contrato sellado y rubricado por el Sindicato Vertical -que era el único que había entonces- y a partir de ese momento me dieron carné como actor (Millás, 2005: 37).

Pero, a pesar de que Luis Prendes contara solamente con tres integrantes del equipo artístico original, el estreno del TEU en el Teatro Español supuso un trampolín para muchos de ellos. Margarita Mas, Blanca Sendino, Pilar Calabuig, Javier Domínguez, Antonio Jiménez y Francisco García comenzaron entonces su andadura profesional; pero sobre todo Juanjo Menéndez (en el papel de Dionisio), José María Prada (como Don Rosario) y Fernando Guillén (como El 
Anciano Militar) comenzaron aquí una carrera que les llevaría a lo más alto del panorama teatral español. Diez de los dieciséis integrantes del reparto original dieron el salto al teatro profesional a raíz del éxito de Tres sombreros de copa. Especialmente chocante resulta, sin embargo, que la protagonista femenina, Gloria Delgado, no accediera al mundo profesional, a diferencia de la mayoría de sus compañeros.

En cuanto al escenógrafo, Emilio Burgos, y la coreógrafa, Karen Taft, ya eran profesionales de reconocido prestigio cuando abordaron el trabajo de esta obra, lo cual da la idea del nivel artístico del teatro universitario del momento. A Agustín González le tocó el papel de Don Sacramento, un rol secundario, pero no para él, que considera que «es una escena formidable, una sola escena, pero es un papel protagonista» (Millás, 2005: 50).

\section{3: la transición al mundo profesional}

González pasó de tres estrenos del TEU en 1952 a siete estrenos (cuatro de ellos con el TEU), una sustitución y una reposición al año siguiente, repartiendo su trabajo entre Barcelona y Madrid. Así, el 11 de marzo estrena en el teatro María Guerrero El momento de tu vida de William Saroyan, donde coincidió con Fernando Guillén nuevamente, y ¡con Francisco Ruiz Ramón!

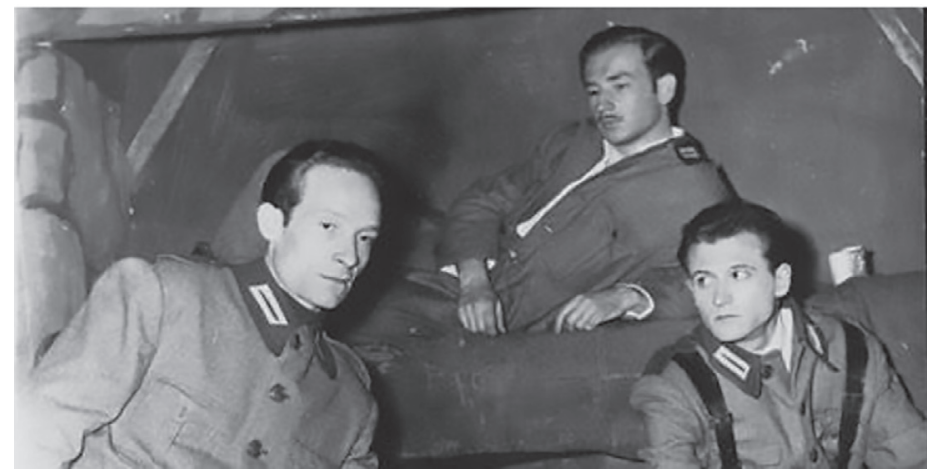

Siete días más tarde, y cuatro meses después del histórico estreno de Tres sombreros de copa, tuvo lugar el de Escuadra hacia la muerte de Alfonso Sastre. Sucede el miércoles 18 de marzo de 1953 en el teatro María Guerrero de Madrid. Las circunstancias que posibilitan el estreno de un texto tan marcadamente anti militar en la etapa franquista son la personalidad del director del María Guerrero, Luis Escobar, sobre el que ya hemos hablado, y su encuadre dentro del TPU, bajo la dirección de Gustavo Pérez Puig. De esta manera lo manifiesta nuestro actor: 
Lo cierto es que se estrenó esta obra de Alfonso Sastre, un disidente del régimen que estaba descaradamente mal visto por parte de las instancias oficiales. En cuanto a la prensa y a otros niveles, no tenía el rechazo brutal de las instituciones gubernamentales. Me acuerdo de que, con motivo de este estreno, se había levantado una gran expectación entre los profesionales del teatro, dotados de un olfato especial para este tipo de cosas, y que, por otra parte, sabían del trabajo que realizaba Alfonso Sastre en agrupaciones como la de Arte Nuevo en el Infanta Beatriz a finales de los años cuarenta. El caso es que Escuadra hacia la muerte resultó un éxito clamoroso y también que, automáticamente, fue prohibida su representación por el ministro del Ejército, Agustín Muñoz Grandes (Millás, 2005: 29-30).

Aun así, Sastre habla de tres representaciones llevadas a cabo por el TPU (1954: 173), y lo cierto es que, al día siguiente del estreno, aparecieron sendos artículos en Arriba y ABC. Este último, firmado por Antonio Rodríguez de León, comienza en estos elogiosos términos: «Estamos por asegurar que Alfonso Sastre acaba de ingresar, tranquila y arriscadamente, en la nómina de los autores españoles de hoy» (1953: 33).

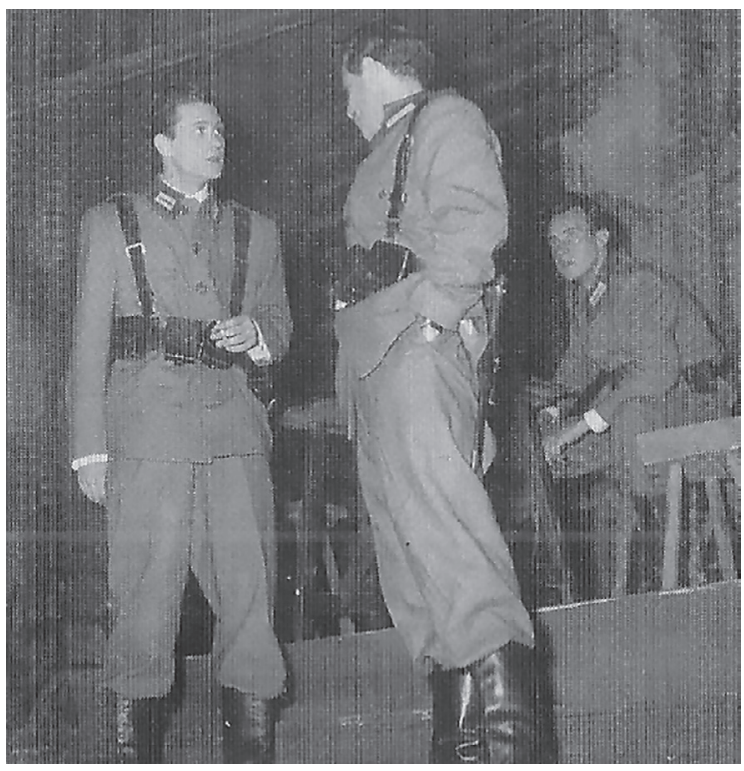

La mitad del reparto lo conforman actores que estrenaron Tres sombreros de copa con el TEU de Madrid. A saber: Agustín González, Juanjo Menéndez y Fernando Guillén. Y, de los otros tres, cabe destacar la figura de Adolfo Marsillach, admirado por González, que se expresa sobre él en estos términos: 
Ya venía precedido de cierto prestigio por los trabajos realizados en Barcelona, era hijo del periodista y crítico de teatro Luis Marsillach. Al llegar a Madrid, también cosechó sus éxitos, sobre todo en la segunda obra de Buero Vallejo, En la ardiente oscuridad, estrenada después de Historia de una escalera. Pero él tenía, además, la ilusión de hacer cosas fuera de la programación del teatro en el que estaba contratado y por esta razón aceptó participar en trabajos en los que, fácilmente, te pasabas ensayando un mes o mes y medio para hacer al autor prohibido o casi prohibido, la función de riesgo que valía la pena (Millás, 2005: 30-31).

Esto no sólo relaciona los sucesos fundamentales de la historia del teatro español de la posguerra, sino que nos da una idea de la importancia que tuvo el TEU en la renovación del panorama teatral del momento. Agustín González interpretó a Adolfo, de entre los seis soldados, «uno de los más malos», según él (Millás, 2005: 50).

Un mes después de Escuadra hacia la muerte, estrena Tres ventanas, de Luis Delgado Benavente, el miércoles 22 de abril en el teatro María Guerrero de Madrid: una puesta en escena del TPU dirigida por Salvador Salazar. Seguidamente, estrena Una bomba llamada Abelardo el miércoles 6 de mayo en el mismo teatro: otra producción del TPU, dirigida esta vez por Gustavo Pérez Puig. Y a partir de entonces, ya sólo se dedicaría al teatro profesional con la salvedad (anecdótica, pero significativa) del reestreno de Tres sombreros de copa al año siguiente. «Fue entonces cuando se formó una compañía en Barcelona para hacer Tres sombreros de copa, y allí fuimos. En esa compañía trabajábamos Fernando Guillén, Mercedes Muñoz Sampedro, su hija Carmen Lozano, Alejandro Maximino, Margarita Lozano y el primer actor, Pablo Garsabal» (Millás, 2005: 38).

Como quiera que fuese, el viernes 14 de agosto estrenaba iiBlum!!, de Enrique Santos Discépolo y Julio Porter en el teatro de la Comedia de Madrid, con la compañía de Marcelino Ornat, primer estreno enteramente profesional de González. Le siguió El café de las flores, de Víctor Ruiz Iriarte, dirigida por Fernando Granada para la compañía de Tina Gascó, y estrenada el viernes 9 de octubre en el teatro Reina Victoria de Madrid. Aún le dio tiempo para hacer una sustitución en Legítima defensa, de Paolo Leví, para la compañía de Carlos Lemos, dirigida por Huberto Pérez de la Ossa en el teatro Beatriz. Y finalizó el año con Federica de Bramante o las florecillas del fango, de Antonio de Lara Tono y Jorge Llopis, en el teatro Beatriz, dirigida también por Pérez de la Ossa, y estrenada el martes 22 de diciembre. González experimentó por primera vez con esta obra el fracaso, pues llegó un día en que no se presentó ni un solo espectador. 
Le dijimos a Carlos Lemos que, puesto que era el primer actor, que tomara una determinación al respecto. Él no quería arrojarse ninguna responsabilidad y dijo que, aunque no hubiera nadie, estaba dispuesto a representar la función, lo que al resto de actores nos parecía bastante descabellado. Para salir del atolladero, a alguien se le ocurrió ir al bar que estaba enfrente del teatro Infanta Beatriz, El Aguilucho, que en gran medida vivía del público del Beatriz, [...] Como en aquella ocasión no acudió nadie a la función, tampoco había clientes en el bar, y fue entonces cuando a alguien se le ocurrió invitar a los camareros, que estaban allí todos sentados con la servilleta colgada del brazo, aburridos y más solos que la una. Echaron el cierre, se sentaron en un palco y, ese día, hicimos la representación para ellos (Millás, 2005: 60).

\section{4: la despedida del TEU}

En este año, el actor realiza cinco estrenos de teatro profesional, su primera incursión en el cine y un único y último montaje universitario: el reestreno de Tres sombreros de copa con el TPU. Interviene en la película Felices Pascuas de Juan Antonio Bardem, y estrena La casa de la noche, de Thierry Maulnier, La eterna doña Juana, de Julia Maura, Eva sin manzana, de Jaime de Armiñán, Irene o el tesoro, de Buero Vallejo, y La mordaza, de Sastre, todas con posterioridad al reestreno de Tres sombreros de copa. De todas estas obras, cabe destacar la puesta en escena de La mordaza, estrenada el 17 de septiembre en el teatro Reina Victoria de Madrid, con dirección de José María de Quinto, y en la que colabora con Fernando Guillén y María Luisa Ponte, que sería su compañera durante más de treinta años.

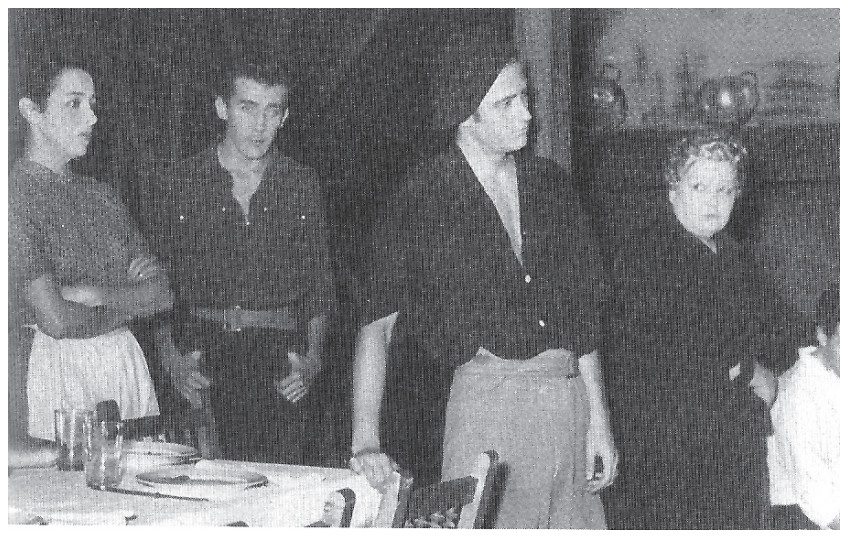

En el reestreno de Tres sombreros de copa por el TPU, repiten el director, Gustavo Pérez Puig, el escenógrafo, la coreógrafa y nueve de los dieciséis actores originales, entre ellos Juanjo Menéndez, José María de Prada y Agustín González. 
No deja de ser significativo que González se despida del teatro universitario con la misma obra que le otorgó el reconocimiento de la crítica y el público y que cambió para siempre su carrera y su vida.

Los años de Agustín González en la universidad fueron determinantes para su formación como actor. Él mismo se consideraba un autodidacta porque sólo había cursado los estudios de bachillerato y comienzo de universidad, pero su afán de conocimiento y de curiosidad había sido tan grande que, según afirmaba, siempre siguió buscando hasta sus últimos días (Millás, 2005: 80). Nunca intentó aplicar unas técnicas determinadas.

Desde la primera vez que pisé un escenario, el teatro lo hago saliendo por las buenas a ver qué pasa y sin plantearme cuestiones dogmáticas. A lo sumo, lo que he hecho ya antes de ser un profesional, es ir al teatro para aprender. He visto a actores y actrices que me impresionaron, y luego, dentro de la práctica de esta profesión, me he parado a ver cómo actuaban los compañeros y he sacado conclusiones (Millás, 2005: 121-122).

Así pues, se puede decir que la escuela de Agustín González fue la experiencia profesional, que no tuvo una formación específica. El teatro universitario se constituyó entonces en un vivero de profesionales escénicos, y un hervidero de creatividad y renovación sobre el que Alfonso Sastre planteó, desde su propia experiencia en él, la siguiente reflexión crítica:

La Universidad está necesitada de un Teatro en el que resuenen experimentalmente las voces de la cultura que, en un severo plano teórico, suenan en las aulas; y que el Teatro está necesitado de la Universidad. Esto segundo porque: 1. ${ }^{\circ}$ Es inaceptable que el Teatro, que es un importante instrumento social, se encuentre íntegramente en las manos de gente torpe, indocta, egoísta, inmoral. Esto hace precisa la incorporación al Teatro de la gente docta y honesta que puede y debe dar la Universidad. 2. ${ }^{\circ}$ Es inaceptable que la educación dramática de las nuevas generaciones se realice caóticamente en el seno de las compañías constituidas y en el transcurso de las giras por los pueblos: el teatro queda así confundido con la picardía; y el noble oficio de actor exige la degradación de un comienzo en el que los que verdaderamente podrían ser los maestros son inaccesibles. Esto podría remediarse organizando la enseñanza del teatro según una estructura universitaria.

Parece ser que esto — que la Universidad venga en auxilio del Teatroes lo que se ha pretendido con la creación de la Escuela Superior de Arte Dramático, que ha empezado a funcionar en Madrid en 1953. Se trata, o por lo menos debería tratarse, de que desaparezca la insuficiente y anacrónica «Sección de Declamación» del Conservatorio. No se trata, naturalmente, de dar un nuevo nombre y un nuevo local a la vieja «Sección de Declamación» (173-174).

Anales, 29-30 (2018), pp. 119-132 
De este modo, se podría afirmar que el panorama de la formación teatral desde el final de la Guerra Civil hasta la segregación de la Escuela Superior de Arte Dramático de Madrid a partir del Real Conservatorio de Música y Declamación, se dividía entre los usos anacrónicos de este conservatorio y la práctica escénica profesional que el régimen franquista permitía (igualmente anacrónica), de manera que la necesidad de renovación vino a ser cubierta por el TEU, que, en tiempos de carestía, contó con la financiación, la estructura y el apoyo indirecto del régimen. Sin embargo, esta renovación estética y dramatúrgica no fue acompañada de una formación técnica adecuada para los artistas que comenzaban en el TEU su andadura profesional. Cuánto mejor no hubiera sido que Agustín González y toda su generación hubieran podido recibir la formación técnica que, en esos mismos años, ya existía en Estados Unidos, Gran Bretaña, la URSS, etc. Aún hoy, ese encaje del teatro en la universidad y de la universidad en el teatro cuya posible solución veía Sastre en 1954 con la «creación» de la Escuela Superior de Arte Dramático, sigue siendo una asignatura pendiente, sobre todo tras la resolución de 2012 del Tribunal Supremo que anula la legitimidad de las escuelas superiores de arte dramático para expedir el título de grado. ${ }^{3}$

Pero volviendo a los años de Agustín González en el TEU de Madrid, aparte de la universidad, el teatro, el cine, Madrid y Barcelona, tuvo que compaginar su actividad artística con el servicio militar, que desempeñó en las oficinas del Ministerio del Ejército en Madrid. Por las mañanas servía en las oficinas del ejército, por las tardes ensayaba y por las noches actuaba.

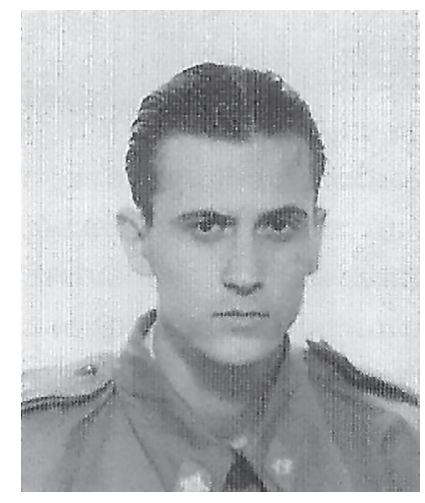

3. Tribunal Supremo, Sala Contencioso-Administrativo, Sentencia 348/2012. 
Allí, en aquel extraño lugar, yo era como Jesucristo entre los doctores, era el amo. Aquellos soldados, que eran unos tipos estupendos, me escuchaban arrobados porque para ellos el hecho de que yo hablara de libros y que trabajara en el teatro constituía algo extraordinario. [...] pasado el primer año, mi ascendiente en esa oficina era tal, que hacía lo que me daba la gana, incluso llegó un momento en que iba vestido de paisano y el capitán y el teniente me trataban con deferencia por el hecho de que me codeara con actores (Millás, 2005: 66-67).

Como queda dicho, Agustín González contribuyó a la labor del TEU como renovador del panorama teatral. Labor que fue reconocida después en la declaración de las I Jornadas de Teatro Universitario de Murcia de 1963: «2. Consideramos que el teatro en España no sirve al momento histórico en que vive, siendo absolutamente imprescindible una renovación teatral que sólo la Universidad está en condiciones de realizar» (Pérez-Rasilla, en García Lorenzo, 1999: 48). Además, fue un componente representativo del colectivo, que buscó y halló en el TEU el paso al teatro profesional. Esta actitud, criticada por Hormigón como «una búsqueda de un individualismo existencial» (1974) y admitida por la reunión de Murcia, para la que «El paradigma y horizonte de aquellos elencos era el teatro profesional» (Oliva, en García Lorenzo, 1999: 15-30), es defendida por Alfonso Sastre en estos términos:

El teatro universitario es, y está bien que así sea, un lugar de paso: un lugar de estudio y de preparación, donde el futuro hombre de teatro hace sus primeras armas, con todas sus consecuencias, ante un público que desde entonces puede seguir su trabajo o rechazarlo (1954: 173-174).

Es habitual que se menosprecie la labor de los actores considerándola como de segundo orden, sobreentendiendo que la elección de los textos, su adaptación y puesta en escena es labor exclusiva del director. En ese sentido, sólo se reconoce el trabajo de Salvador Salazar, Gustavo Pérez Puig o Alfonso Sastre en la renovación del panorama teatral español desde el TEU. Pero en el caso de Agustín González, no sólo hay que reconocer su intervención como actor en un arte que es necesariamente colectivo, sino que además hay que poner de relieve su parte de responsabilidad en la línea filosófica y social de la generación de la que hablamos, puesto que formó parte del Grupo de Teatro Realista junto con Alfonso Sastre y José María de Quinto, y eligió participar en espectáculos de teatro independiente que sólo podían traerle problemas con el régimen.

En resumen, el TEU supuso para Agustín González el comienzo de su carrera, el éxito, y la participación privilegiada en los principales acontecimientos de la historia del teatro español de la posguerra. 


\section{Bibliografía citada}

Hormigón, Juan Antonio, Teatro, realismo y cultura de masas, Madrid, Cuadernos para el Diálogo, 1974.

Millás, Lola, Agustín González. Entre la conversación y la memoria, Madrid, Ocho y medio, 2005.

Oliva, César, «La escena universitaria española», en L. García Lorenzo (ed.), Aproximación al Teatro Español Universitario (TEU), Madrid, CSIC, 1999, pp. 15-30.

PÉREZ-RASILLA, Eduardo, «La situación del teatro universitario en España desde 1939 a 1967», en L. García Lorenzo (ed.), Aproximación al Teatro Español Universitario (TEU), Madrid, CSIC, 1999, pp. 31-53.

Rodríguez de LEÓn, Antonio, «Cartelera madrileña de espectáculos», en $A B C$, 19 de marzo de 1953.

RPdrígueZ PAdrón, Jorge, Pról. a M. Mihura, Tres sombreros de copa, Madrid, Cátedra, 1997.

SASTRE, Alfonso, «Los teatros universitarios en 1953», Revista de Educación, 17 (1954), pp. 173-174. 